\section{Reasons for leaving home}

\author{
Paul Harvey
}

Mammalian Dispersal Patterns: The Effects of Social Structure on Population Genetics. Edited by B. Diane ChepkoSade and Zuleyma Tang Halpin. University of Chicago Press:1987. Pp.342. Hbk $\$ 55, £ 43.95$; pbk \$19.95, £15.95.

TyPiCAlLy, male mammals leave their place of birth in order to breed, while the female stays at home if social conditions allow. In contrast, among most bird species the females move farther than the males. The reason for these opposite patterns in the two groups is, to my mind, a mystery. Behavioural studies of known individuals from several species indicate that incest avoidance is often involved. If that is the case, only one sex need move. But why should it be males in mammals and females in birds? The various theories based on whether the breeding system is one of resource or mate defence have never convinced me, primarily because they do not explain the exceptions without a good deal of special pleading.

The facts of sex-biased and limited dispersal in mammals are both the starting point and the crux of Chepko-Sade and Halpin's edited volume. The subtitle of the book, The Effects of Social Structure on Population Genetics, could cover a multitude of sins. So what exactly are the editors after? If one number encapsulates their Grail it is $N_{\mathrm{e}}$, Sewall Wright's effective or neighbourhood population size. As the editors write in their preface (p.xiv), they are interested in the usefulness of $N_{\mathrm{e}}$ for "characterizing and comparing populations and predicting the relative importance of Wright's shifting balance theory in the tempo and mode of evolution". It is not surprising that the Quest turned out to be of more value than the End.

The quality of data on dispersal emerging from long-term studies of mammals is improving. This volume brings together a representative sample of detailed descriptive but non-genetic work on known individuals from deer, horses, wolves, bears, mongooses, prairie dogs, kangaroo rats and pikas. Data from these studies are used in the final chapter to estimate $N_{\mathrm{e}}$, which generally turns out to be surprisingly small (often tens rather than hundreds of individuals). The editors and their coauthors warn of the tentative nature of their estimates, but conclude that "the shifting balance process is likely to occur very effectively in ... horses, mongooses, ... prairie dogs,

kangaroo rats and ... pikas" (p.314) They also consider the "numerous genetic consequences" (p.314) that their analyses

imply, including the importance of genetic drift and a high rate of inbreeding.

In an earlier chapter, however, Alan Templeton challenges these results when he points out that the levels of inbreeding they imply would eliminate inbreeding depression. The near universal occurrence of inbreeding depression in studies of captive populations of mammals seems to contradict this prediction. Templeton concludes that the estimates of effective or neighbourhood population size are probably incorrect because assumptions of the models are not fulfilled. As he points out, a clearer picture will probably emerge when we have accurate estimates of paternity (genetic fingerprinting should see to that), when we find whether recolonizations following local extinctions are from one or several neighbouring groups, and when we get a clearer indication of

\section{Fast forward}

\section{Frederick James}

Collider Physics. By Vernon D. Barger and Roger J. N. Phillips. Addison-Wesley: 1987. Pp.592. \$44.95, £29.95.

IT COULD be argued that collider physics as an isolated subject does not exist, and Barger and Phillips here contribute to that argument by including a considerable amount of material well outside the realm indicated by their title. The result is a relatively self-contained text on modern particle physics suitable for advanced graduate students and young research workers. Given the rapid advances in this field in recent years, especially on the theoretical side, I suspect that more than one established physicist will also benefit from the coherent (although necessarily dense) presentation and the emphasis on up-to-date topics as studied in current and near-future colliders.

The text was computer-prepared using $\mathrm{T}_{\mathrm{E}} \mathrm{X}$, and is well laid out, but because of the relatively large typeface there is not as much of it as one would expect in 592 pages. The index is good enough to make the book also handy as a reference work.

One useful aspect of the book is the large number of derivations of expressions for matrix elements corresponding to particular Feynman diagrams. These are not just a help in understanding the physics, but also provide the working physicist with a good starting point for standard model calculations.

On the other hand, the experimentalist will not be fooled by the attempt to make the coverage appear comprehensive by inclusion of a few pages on detectors and the colliders themselves: the book is resolutely orientated towards the theoretical side. This is hardly surprising because the whether human disturbance has led to recent subdivision of populations being studied.

In addition to the chapters I have mentioned, there are others on the genetic structure of small mammal populations, on dispersal and demography in two human populations, and on the evolution of kin selection in complex groups. But it is because the editors have pushed the field data to their limits that this book is most valuable. Those with ideas about the causes and consequences of dispersal in natural populations will be helped to formulate their own questions in terms of the available data. More often, though, they will find that the necessary information has yet to be collected.

Paul Harvey is in the Department of Zoology, University of Oxford, South Parks Road, Oxford OXI $3 P S$, UK.

authors are theoretical physicists, and one would not expect a complete treatment of experimental problems, but some topics of special interest to experimentalists are skipped over too lightly. For example, only a few lines are devoted to Bhabha scattering, and expressions for cross sections for polarized electrons are given without any comment on the problems of obtaining polarized beams or on the importance of polarization for the measurement of physically interesting quantities.

The chapter on Monte Carlo simulations includes listings of several Fortran programs. I applaud this growing practice Fortran is a widely understood language and a good vehicle for the precise expression of certain ideas, becoming in fact the scientific equivalent of a French or Latin quotation in a history book. Readers should not however take literally the authors' claim that the programs are written according to the Fortran 77 standard, because they contain some constructs (such as lower-case characters and REAL $* 8$ declarations) which are not part of the standard and are not accepted by several popular compilers.

Barger and Phillips are, of course, at their best in the chapters devoted to the more speculative theoretical developments: entire chapters are devoted to heavy quark production, the Higgs boson(s), the fourth generation and the various higher symmetries. This is after all what collider physics is all about, and we have here about as clear a snapshot of this fast-moving subject as the circumstances allow. In these chapters the authors have chosen to concentrate on the most promising classes of theories rather than particular models. We should know within a few years whether their choice was judicious.

Frederick James is in the Data Handling Division at CERN, 1211 Geneva 23, Switzerland. 